\title{
A Parallel Multirate Algorithm for the Numerical Integration of System of Nonlinear Differential Equations
}

\author{
Petro Stakhiv ${ }^{1)}$, Serhiy Rendzinyak ${ }^{2}$ \\ 1) Prof., D.Sc. Eng., St. Bandera 12, 79013 Lviv UKRAINE, spg@polynet.lviv.ua \\ 2) Ass. Prof., Dr. Eng., St. Bandera 12, 79013 Lviv UKRAINE
}

\begin{abstract}
The new approach to calculate dynamic behavior of large-scale systems, separated on subsystems is presented. Parallelization efficiency of computing process is described.
\end{abstract}

Keywords: parallel algorithm, multirate method, large-scale systems, nonlinear differential equations.

\section{INTRODUCTION}

Solution of complex systems of differential equations using numerical methods is connected with some computation problems. In order to solve this problem the most suitable methods should be based on decomposition of complex system to more simple parts. It makes possible to share computational resources for more simple tasks. We will not discuss the question of formation of such a system. We consider that it is possible to obtain a system in which each group of state variables is concentrated in some subsystem of differential equations using additional constrain variables.

Unlike to well-known multirate method [1] (the peculiarity of it difference scheme lies in fact that separate subsystems have their time scale) we propose combined multirate relaxation method where each subcircuit can have different physical nature and therefore should be described by different mathematical models $[2,3]$. On the basis of this new method it is possible to create an algorithm with structure which will make it possible to use advantages of parallel computing systems [4].

\section{BASES OF MULTIRATE RELAXATION METHOD}

Integration of subsystems with separate step can be considered as a basis of multirate integration method of subcircuits. It is possible to take into account dynamic features of each subcircuit. In other words in analyzed circuit some subsystems with time latency can exist, so values of their internal and external variables can be practically constant on some time intervals. Besides, some nonlinear subsystems can demand smaller division of integration step then other subsystems in order to ensure the convergence of iterations. In such a case the program for simulation can be run faster.

Let us consider mathematical model of some physical object (previously decomposed into separate parts) in the basis of state variables:

$$
\begin{aligned}
& \vec{F}_{i}\left(\frac{d \vec{x}_{1}}{d t}, \vec{x}_{1}, \vec{u}\right)=0 \\
& G\left(\vec{x}_{1}, \ldots, \vec{x}_{i}, \ldots, \vec{x}_{N}, \vec{u}\right)=0
\end{aligned}
$$

where $F_{i}$ are nonlinear vector-functions (subsystem state equations); $i=\overline{1, N}, \quad N$ is number of subsystems; $G$ is nonlinear function of subsystems coupling; $\frac{d \vec{x}_{i}}{d t}, \vec{x}_{i}$ are vectors of state variables and their derivatives of $i$-th subcircuit; $\vec{u}$ is a vector of external variables.

The essence of multirate integration algorithm lies in independent integration of separate parts of the whole system on some time interval. The each iteration contains two consecutive steps: integration of equations of separate subsystems (1) and coordination of solutions obtained on previous stage using solving of coupling equations (2).

Step of integration for each splitted subsystems can be chosen automatically with consideration of changes of it internal parameters. After integration of all subsystems on one step forward the maximum step can be estimated for $k$-th time interval $H K_{k}=\max h_{i, k}$. All other subcircuits are integrated until time interval will be equal to 
$T_{k}=T_{k-1}+H K_{k}$.

Algorithm of integration of system of nonlinear differential equations using multirate integration method consists of the following steps:

1. Using given initial conditions of state variables $\vec{x}_{i, 0}$ a value of external variables vector $\vec{u}_{0}$ can be calculated and each subsystem should be solved "backward" into negative time domain using explicit Euler's method. Largest integration step of subsystem $h_{i, 0}$ is considered as a step of correction $H K_{0}$. In the moment of time $T_{-1}=T_{0}-H K_{0}$ a value of vector $\vec{u}_{-1}$ is estimated again. Let us consider that number $k$ of next integration point is equal to 1.

2. We integrate all subsystems on one step forward by approximation of external variables vector values $\vec{u}_{k}^{(0)}$ on two values in previous time intervals.

3. Largest integration step of subsystem $h_{i, k}$ is considered as step of subcircuits correction $H K_{k}$.

4. We integrate again all subsystems where $h_{i, k}<H K_{k}$ until time will be equal to $T_{k}=T_{k-1}+H K_{k}$.

5. In the moment of time $T_{k}$ values of external variables vector are estimated $\vec{u}_{k}^{(1)}$, in other words they are corrected using formulas (2). We consider that at this point first simple iteration by external variables is finished. Let us sign its number as $J=1$.

6. If obtained values of parameters of subsystem external variables vector differ from approximated values on more then given value we carry out repeated calculation of all sybsystems on time interval $\quad\left[T_{k-1}, T_{k-1}+H K_{k}\right]$, taking into consideration new values of vector $\vec{u}_{k}^{(J)}$. When number of iterations exceeds the maximum given value we reduce a step of correction compulsorily to value $H K_{k}^{*}$.

7. We carry out transition to execution of point 2 and calculate system on the following time interval $\left[T_{k-1}, T_{k-1}+H K_{k}^{*}\right]$.

In the figure 1 correlation between steps of integration of separate subcircuits and steps of correction during the process of calculation.

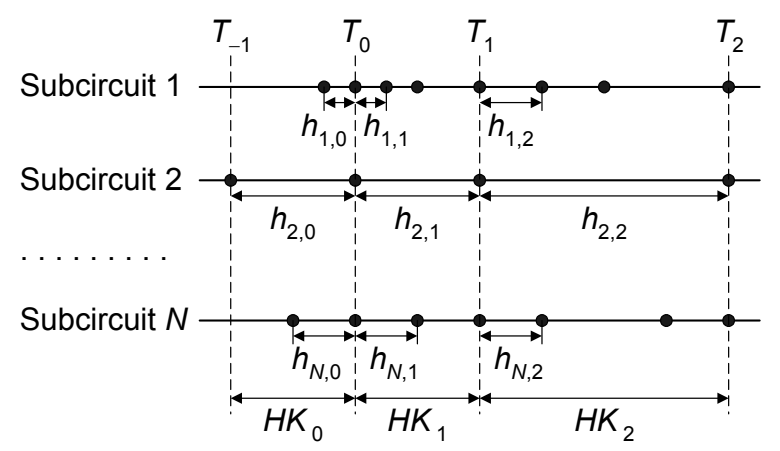

Fig. 1. Algorithm to choice the step of correction of external variables

During implementation of multirate method due to operations carried out with matrices of lower dimensions on common series computation systems a considerable decreasing of computation time can be seen. But this method of integration can loose convergence during analysis of transient processes in subsystems when influence of external variables prevails on state variables of subsystems. Week convergence of multirate method can be explained by presence of component of iteration algorithm of implicit type. It can be explained by fact that calculation of interconnections between subsystems in correction points can be done using explicit scheme using simple iteration method.

\section{PARALLELIZATION OF MULTIRATE METHOD}

In multirate method it is possible to solve each subsystem without dependence on another subsystem by replacement of their influence with the help of additional external variables. In other words a parallelism of calculation of such subcircuits takes place.

Let us consider a system of linear differential equations presented in the following form:

$$
\frac{d \vec{x}}{d t}=\mathbf{A} \vec{x}+\mathbf{B} \vec{v}
$$

where $\vec{x}$ is $n$ - dimensional vector of input variables; $\vec{v}$ is $m-$ dimensional vector of disturbances; $\mathbf{A}$ is a square matrix with dimension $n \times n ; \mathbf{B}$ is a rectangular matrix with dimension $n \times m$.

After the vector of additional variables $\vec{u}$ is introduced into equation (3) it can be rewritten in the following form 

a) $\frac{d \vec{x}}{d t}=\mathbf{P} \vec{x}+\mathbf{B} \vec{v}+\mathbf{D} \vec{u}$
b) $\mathbf{C} \cdot\left[\begin{array}{l}\vec{x} \\ \vec{u}\end{array}\right]=[0]$

A peculiarity of a set of equations (4) is a fact that matrix $\mathbf{P}$ has a block-diagonal structure $\mathbf{P}=\operatorname{diag}\left(\mathbf{P}_{1}, \mathbf{P}_{2}, \ldots \mathbf{P}_{l}\right) . \mathbf{P}_{i}, \mathbf{C}$ and $\mathbf{D}$ are matrices of corresponding sizes.

Let us mark that during the mentioned transform a dimension of vector $\vec{u}$ can't be less than $n$ and number of blocks $l$ is less than $n$ evidently.

Taking into account a structure of matrix $\mathbf{P}$ the first equation from the set (4) can be decomposed to $l$ separate matrix equations in the following way:

$$
\begin{gathered}
\frac{d \vec{x}_{i}}{d t}=\mathbf{P}_{i} \vec{x}_{i}+\mathbf{B}_{i} \vec{v}+\mathbf{D}_{i} \vec{u} \\
i=1,2, \ldots, l
\end{gathered}
$$

where $\vec{x}_{i}$ are corresponding subvectors of vector of variables $\quad \vec{x}^{T}=\left[\vec{x}_{1}^{T}, \vec{x}_{2}^{T}, \ldots \vec{x}_{l}^{T}\right] ; \quad B_{i}, D_{i} \quad$ are submatrices of matrices $B$ and $D$ composed from their some rows.

The following difference scheme for solving of a set of algebraic and differential equations (4) is proposed:

$$
\begin{gathered}
x_{i, k+1}=h b_{-1} \mathbf{P}_{i} x_{i, k+1}+h b_{-1} \mathbf{B}_{i} v_{i, k+1}+ \\
+h b_{-1} \mathbf{D}_{i} u_{k+1}+F_{i, k}
\end{gathered},
$$

where $i=1,2, \ldots l ; k=0,1,2 \ldots$ is number of step of integration, $b_{-1}$ is a coefficient which defines implicit method of integration, $F_{i, k}$ is a vector which contains information about state variables in previous integration points. If step $h$ is the same for all values of $i$ then let us add to the set of state equations the following constraint equations

$$
\mathbf{C}\left[\begin{array}{c}
x_{i, k+1} \\
u_{k+1}
\end{array}\right]=0,
$$

we will obtain a system of algebraic equations with block-diagonal form using a frame of direct diakoptic approach. Algorithm to solve such a system is created on the basis of block Gauss method. During forward trace values of state variables $u_{k+1}$ should be calculated, and during reverse trace - values of state variables $x_{i, k+1}$. Numerical effectiveness and acceleration of this method are investigated rather in details in different works, for example in [5].

Using iteration method to solve a set of algebraic equations with block-diagonal form with frame it is possible to improve a numerical effectiveness of some tasks and to increase acceleration of parallel algorithm. Taking into considering inevitable restrictions of convergence and stability of analyzed circuit, adequacy of obtained results greatly depends on decomposition of vector $x$ on subvectors.

Let us use the iteration method of Jacobi

$$
\begin{aligned}
& \left(\mathbf{1}-h b_{-1} \mathbf{P}_{i}\right) x_{i, k+1}^{(j+1)}=h b_{-1} \mathbf{B}_{i} v_{i, k+1}^{(j+1)}+ \\
& +h b_{-1} \mathbf{D}_{i} u_{i, k+1}^{(j)}+F_{i, k+1} \\
& i=1,2, \ldots, l ; k=0,1,2, \ldots \text {. }
\end{aligned}
$$

In general a set of constrain equations can be presented in explicit form:

$$
u_{k+1}^{(j)}=\mathbb{C} x_{i, k+1}^{(j)} .
$$

As it can be seen, due to selected approach of discretization obtained system of equations (6-7) is decomposed into $l+1$ separated subsystems of linear equations which can be solved independently one from another. During this process subsystems of equations (6) are solved first, and subsystems (7) after them.

Inherently proposed difference system is a combined (explicit-implicit) method of numerical integration of system of differential equations where variables $\vec{x}$ are integrated by implicit method and variables $\vec{u}$ by explicit method.

Known drawbacks of such numerical methods are caused by their potential instability. Because of this allowed value of step of integration is rather small. But at the same time these methods have essential advantages connected with possibilities to integrate with variable step different groups of variables and to create on their basis parallel algorithms of numerical integration intended for large systems of differential equations. And, of course, it is important that combined methods are more accurate potentially.

It is not difficult to spread proposed method on systems of nonlinear differential equations which can be written down in the following form:

$$
\frac{d \vec{x}}{d t}=\vec{F}(\vec{x}, \vec{v}),
$$


where $\vec{F}(\cdot)$ is some nonlinear vector-function of many variables.

Similarly, to linear case we introduce additional variables which in general are linear combination of variables $\vec{x}$ :

$$
\begin{aligned}
& u_{k}=\sum_{i=1}^{n} \alpha_{k i} x_{i}, \\
& k=1,2, \ldots n,
\end{aligned}
$$

where $\alpha_{k i}$ are some constant coefficients.

When vector of additional variables is chosen correctly a system of equations can be reduced to the form

$$
\begin{aligned}
& \frac{d \vec{x}_{i}}{d t}=F_{i}\left(\vec{x}_{i} \vec{v} \vec{u}\right), i=1,2, \ldots, l, \\
& \mathbf{C} \cdot\left[\begin{array}{c}
\vec{x} \\
\vec{u}
\end{array}\right]=[0], \vec{u}=G(\vec{x}),
\end{aligned}
$$

which will have the following difference scheme:

$$
\begin{aligned}
& \vec{x}_{i}^{(k+1)}=\vec{F}_{i}^{(k)}\left(\vec{x}_{i}^{(k+1)} \vec{v}^{(k+1)} \vec{u}^{(k)}\right), i=1,2, \ldots, l,(10) \\
& \mathbf{C} \cdot\left[\begin{array}{l}
\vec{x}^{(k+1)} \\
\vec{u}^{(k+1)}
\end{array}\right]=[0], \vec{u}^{(k+1)}=G\left(\vec{x}^{(k+1)}\right),
\end{aligned}
$$

here $\vec{F}_{(1)}^{T}=\left[\vec{F}_{1(.)}^{T} ; F_{2(.)}^{T} ; \ldots ; F_{l(.)}^{T}\right]$.

Generally in nonlinear case in order to solve nonlinear equations (10) respectively to variables $\vec{x}_{i}$ it is necessary to use some iteration procedures at each step. That fact makes a process of equations integration more complicated, but possibilities to parallelize computing process are the same as in linear case. Therefore, realization of the parallel algorithm can be carried out for nonlinear system of differential equations [4].

A block scheme of algorithm is shown in the Fig. 2.

\section{EFFIICIENCY OF PARALLEL ALGORITHM}

Numerical effectiveness

$$
E_{\text {num }}=\frac{T_{0}}{T_{1}}
$$

$$
R=\frac{T_{1}}{T_{S}}
$$

depend on $T_{S}$ (run time of parallel algorithm by computer with $S$ processors), $T_{1}$ (run time of parallel algorithm by system with one processor) and $T_{0}$ (run time of series algorithm without considering of parallelism). Evidently that it is enough to define these time characteristics for one step of integration. In general they depend on number of steps of integration, and, hence, on dynamic properties of the whole scheme and separate subsystems.

Let us consider run time of series algorithm during one step of integration as complexity of factorization of a matrix with dimension $n \times n$

$$
T_{0}=O(n)=C\left(a_{0}+a_{1} n+a_{2} n^{2}+a_{3} n^{3}\right)
$$

If subsystems will have approximately the same size equal to rounded integer $n_{l}=n / l$ then run time of the parallel algorithm with one processor is

$$
T_{1}=N_{j}\left[l O\left(n_{l}\right)+m n t_{m}+m(n-1) t_{a}\right],
$$

where $N_{j}$ is number of iterations relatively to variables of coupling, $m$ is number of coupling variables $\vec{u}, t_{m}$ and $t_{a}$ are execution times of multiplication and addition. Conducted calculations have shown that it is enough to carry out the one iteration if the iteration process is convergent. Taking into account a complexity of factorization of the matrix that depends on its size in some program environment a numerical effectiveness of parallel algorithm was evaluated:

$$
E_{n u m}=\frac{O(n)}{l O\left(n_{l}\right)+m n t_{m}+m(n-1) t_{a}} .
$$

During running this task on given computer, the following coefficients were obtained: $C=0.178 \mathrm{~ms}$, $a_{0}=-1, \quad a_{1}=1.44, \quad a_{2}=0.182, \quad a_{3}=0.00842$. Time intervals needed to perform addition and subtraction operations are approximately the same and are equal $t_{a}=t_{m}=86.8 \mathrm{~ns}$. Dependence of numerical effectiveness on number of subsystems and task dimension is shown in the fig. 3 .

and acceleration 


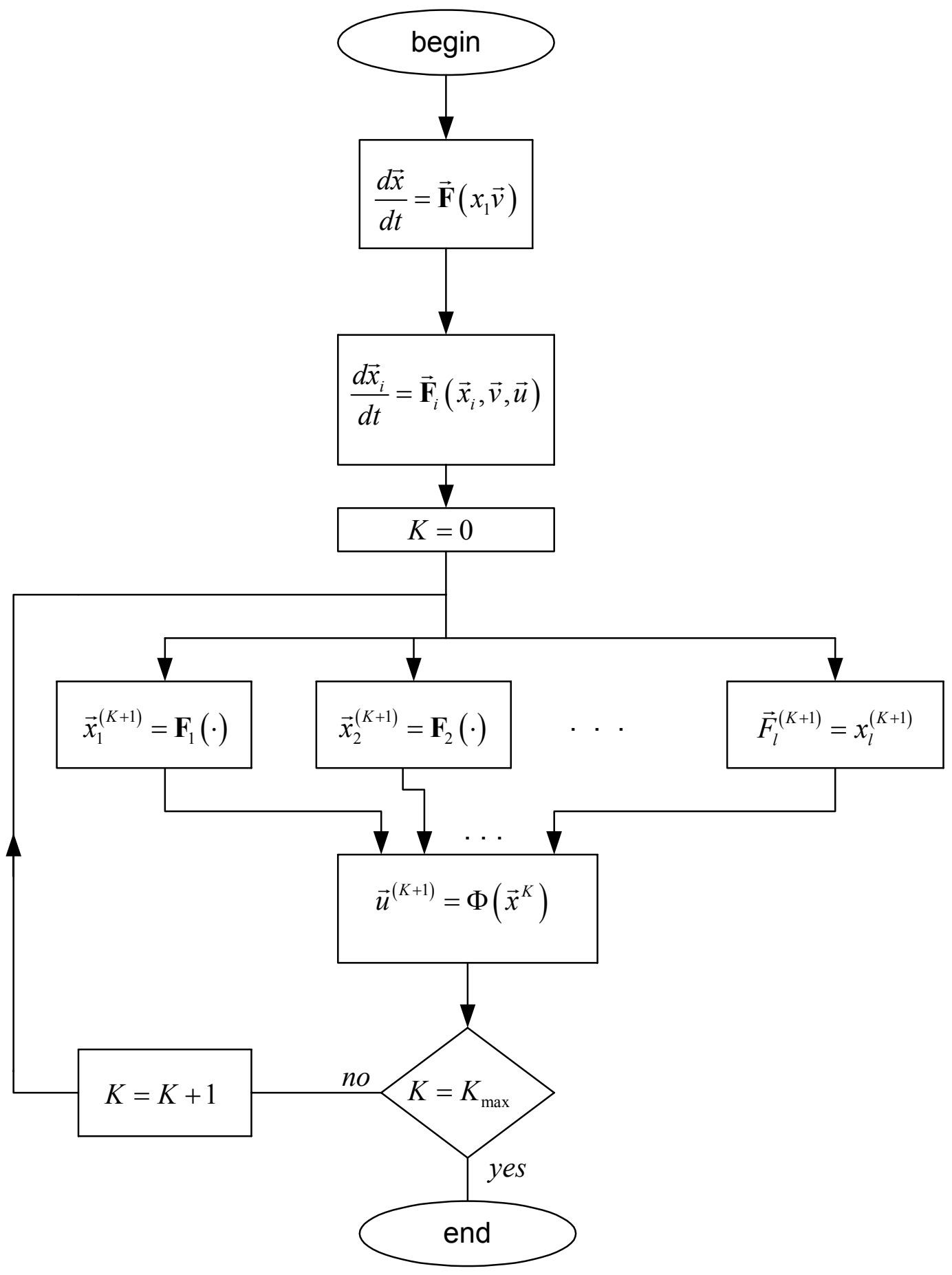

Fig. 2. Block-scheme of computing algorithm



\section{EXAMPLES}

Example 1.

A set of equations of the fourth order is decomposed to two subsystems of the second order [1].

Simulation of transient process of the whole system

Fig. 3. Dependence of numerical effectiveness $E_{\text {num }}$ on number of subsystems $l$ for one step 


$$
\begin{aligned}
&\left(\begin{array}{c}
\dot{x}_{1} \\
\dot{x}_{2} \\
\dot{x}_{3} \\
\dot{x}_{4}
\end{array}\right)=\left(\begin{array}{cccc}
-1 & 0 & 0 & 0 \\
0 & -10 & 0 & 0 \\
0 & 0 & -40 & 0 \\
0 & 0 & 0 & -100
\end{array}\right)\left(\begin{array}{l}
x_{1} \\
x_{2} \\
x_{3} \\
x_{4}
\end{array}\right)+ \\
&+\left(\begin{array}{cccc}
0 & 0 & 0 & 0 \\
0.1 & 0 & 0 & 0 \\
4 & 4 & 0 & 0 \\
1 & 1 & 1 & 0
\end{array}\right)\left(\begin{array}{l}
x_{1}^{2} \\
x_{2}^{2} \\
x_{3}^{2} \\
x_{4}^{2}
\end{array}\right)+\left(\begin{array}{l}
2 \\
0 \\
0 \\
0
\end{array}\right), \\
&\left(\begin{array}{l}
x_{1}(0) \\
x_{2}(0) \\
x_{3}(0) \\
x_{4}(0)
\end{array}\right)=\left(\begin{array}{l}
1 \\
1 \\
1 \\
1
\end{array}\right)
\end{aligned}
$$

is shown in fig. 4.



Fig. 4. Time chart of variables of the whole system

The same circuit decomposed on two subcircuits,

$$
\begin{aligned}
\left(\begin{array}{l}
\dot{x}_{1} \\
\dot{x}_{2}
\end{array}\right)= & \left(\begin{array}{cc}
-1 & 0 \\
0 & -10
\end{array}\right)\left(\begin{array}{l}
x_{1} \\
x_{2}
\end{array}\right)+\left(\begin{array}{cc}
0 & 0 \\
0.1 & 0
\end{array}\right)\left(\begin{array}{l}
x_{1}^{2} \\
x_{2}^{2}
\end{array}\right)+ \\
& +\left(\begin{array}{l}
2 \\
0
\end{array}\right)+\left(\begin{array}{ll}
0 & 0 \\
0 & 0
\end{array}\right)\left(\begin{array}{l}
u_{1} \\
u_{2}
\end{array}\right) ; \\
\left(\begin{array}{l}
\dot{x}_{3} \\
\dot{x}_{4}
\end{array}\right)= & \left(\begin{array}{ll}
-40 & 0 \\
x_{1}(0) \\
x_{2}(0)
\end{array}\right)=\left(\begin{array}{l}
1 \\
1
\end{array}\right) ; \\
& +\left(\begin{array}{ll}
4 & 4 \\
1 & 1
\end{array}\right)\left(\begin{array}{l}
u_{1} \\
u_{2}
\end{array}\right) ;
\end{aligned}
$$

$$
\left(\begin{array}{l}
x_{3}(0) \\
x_{4}(0)
\end{array}\right)=\left(\begin{array}{l}
1 \\
1
\end{array}\right)
$$

with additional variables of coupling

$$
\begin{aligned}
& \left(\begin{array}{l}
u_{1} \\
u_{2}
\end{array}\right)=\left(\begin{array}{llll}
0 & 0 & 0 & 0 \\
0 & 0 & 0 & 0
\end{array}\right)\left(\begin{array}{l}
x_{1} \\
x_{2} \\
x_{3} \\
x_{4}
\end{array}\right)+ \\
& +\left(\begin{array}{llll}
1 & 0 & 0 & 0 \\
0 & 1 & 0 & 0
\end{array}\right)\left(\begin{array}{c}
x_{1}^{2} \\
x_{2}^{2} \\
x_{3}^{2} \\
x_{4}^{2}
\end{array}\right),
\end{aligned}
$$

calculated according to presented algorithm (fig. 5).

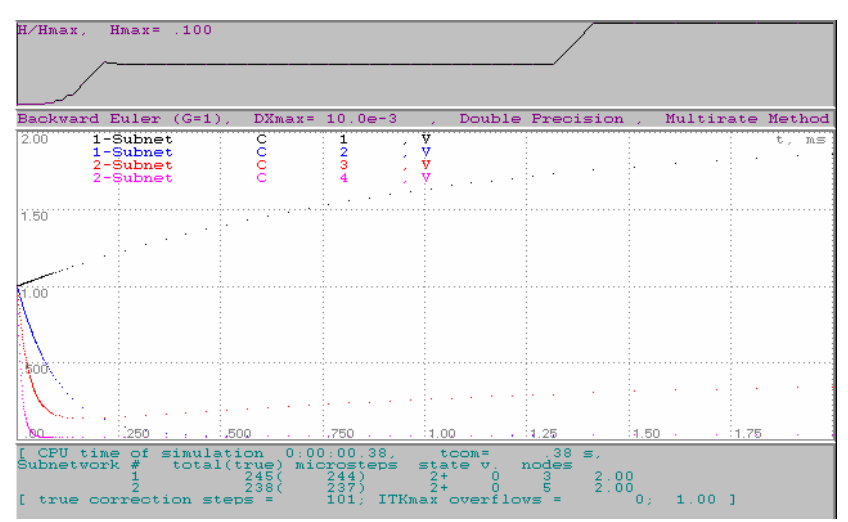

Fig. 5. Time chart of variables of two subsystems

Example 2.

A set of equations of the fourth order is decomposed to two subsystems of the second order.

Simulation of transient process of the whole system

$$
\begin{aligned}
\left(\begin{array}{l}
\dot{x}_{1} \\
\dot{x}_{2} \\
\dot{x}_{3} \\
\dot{x}_{4}
\end{array}\right) & =\left(\begin{array}{cccc}
0.5 & -0.001 & 0 & -0.001 \\
1000 & 0 & 0 & 0 \\
0 & 0 & 1.5 & 0.001 \\
1000 & 0 & -1000 & 0
\end{array}\right)\left(\begin{array}{l}
x_{1} \\
x_{2} \\
x_{3} \\
x_{4}
\end{array}\right)+ \\
& +\left(\begin{array}{cc}
-1 & 0 \\
0 & 0 \\
0 & -1 \\
0 & 0
\end{array}\right)\left(\begin{array}{l}
x_{1}^{3} \\
x_{2}^{3}
\end{array}\right)+\left(\begin{array}{l}
1 \\
0 \\
0 \\
0
\end{array}\right),\left(\begin{array}{l}
x_{1}(0) \\
x_{2}(0) \\
x_{3}(0) \\
x_{4}(0)
\end{array}\right)=\left(\begin{array}{l}
0 \\
0 \\
0 \\
0
\end{array}\right)
\end{aligned}
$$

is shown in fig. 6 . 


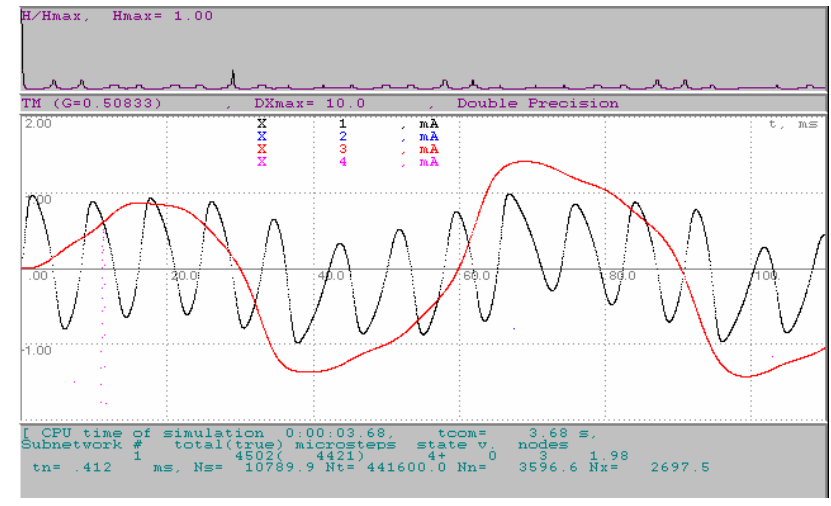

Fig. 6. Time chart of variables of the whole system

The same circuit decomposed on two subcircuits,

$$
\begin{aligned}
& \left(\begin{array}{l}
\dot{x}_{1} \\
\dot{x}_{2}
\end{array}\right)=\left(\begin{array}{cc}
0.5 & -0.001 \\
0 & -10
\end{array}\right)\left(\begin{array}{l}
x_{1} \\
x_{2}
\end{array}\right)+\left(\begin{array}{c}
-1 \\
0
\end{array}\right) x_{1}^{3}+ \\
& +\left(\begin{array}{l}
1 \\
0
\end{array}\right)+\left(\begin{array}{cc}
-0.001 & 0 \\
0 & 0
\end{array}\right)\left(\begin{array}{l}
u_{1} \\
u_{2}
\end{array}\right) ;\left(\begin{array}{l}
x_{1}(0) \\
x_{2}(0)
\end{array}\right)=\left(\begin{array}{l}
0 \\
0
\end{array}\right) ; \\
& \left(\begin{array}{l}
\dot{x}_{3} \\
\dot{x}_{4}
\end{array}\right)=\left(\begin{array}{cc}
1.5 & 0.001 \\
-1000 & 0
\end{array}\right)\left(\begin{array}{l}
x_{3} \\
x_{4}
\end{array}\right)+\left(\begin{array}{c}
-1 \\
0
\end{array}\right) x_{3}^{3}+ \\
& +\left(\begin{array}{cc}
0 & 0 \\
0 & 1000
\end{array}\right)\left(\begin{array}{l}
u_{1} \\
u_{2}
\end{array}\right) ;\left(\begin{array}{l}
x_{3}(0) \\
x_{4}(0)
\end{array}\right)=\left(\begin{array}{l}
0 \\
0
\end{array}\right) ;
\end{aligned}
$$

with additional variables of coupling

$$
\left(\begin{array}{l}
u_{1} \\
u_{2}
\end{array}\right)=\left(\begin{array}{llll}
0 & 0 & 0 & 1 \\
1 & 0 & 0 & 0
\end{array}\right)\left(\begin{array}{l}
x_{1} \\
x_{2} \\
x_{3} \\
x_{4}
\end{array}\right) \text {, }
$$

calculated according to presented algorithm (fig. 7).

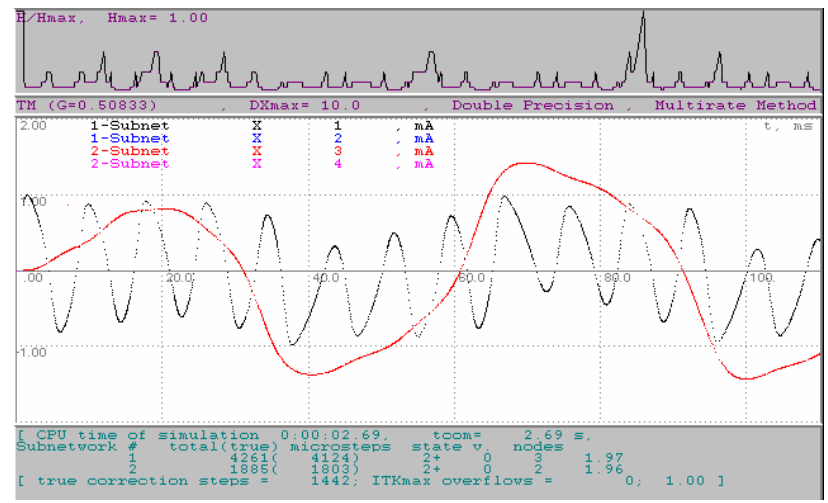

Fig. 7. Time chart of variables of two subsystems

Presented example confirms conclusion that transient process in decomposed system is simulated with lower number of steps of integration, and therefore more effectively.

\section{CONCLUSIONS}

As a result of presented research it is possible to say that the methods of analysis which makes possible to consider dynamic features of subsystems are most important for scientists and engineers.

\section{REFERENCES}

[1] D. Petcu, Parallelism in solving ordinary differential equations, Mathematical Monographs 64, Tipografia Universitatii din Timisoara, 1998, p. 232.

[2] S.Y. Rendzinyak, Efficiency analysis of subcircuit methods of transitional process simulation on some large-scale radio electronic circuits (in Russian), Teoreticheskaya elektrotekhnika, Vol. 40, (1986), pp. 139-143.

[3] P.H. Stakhiv, S.Y. Rendzinyak, P.R. Strubytskyy, Stability of numerical scheme of diakoptic methods of time-domain modeling of large-scale circuits (in Ukrainian), Electronika $i$ sviaz, 1, 3, Vol. 1, (1997), pp. 188-195.

[4] Petro Stakhiv, Serhiy Rendzinyak, Bohdan Krupskyy, Parallelization of Diakoptic Methods for Multiprocessor Computing Systems. Bull. Pol. Ac.: Tech., Vol. 51, No. 3 (2003), pp. 381394.

[5] K.V. Kharchenko, Modification of paralleling block-diagonal solution methods of linear systems of equations for applying to CAD electronic circuits (in Ukrainian), Electronika $i$ sviaz, 1, 6, (1999), pp. 156-162.

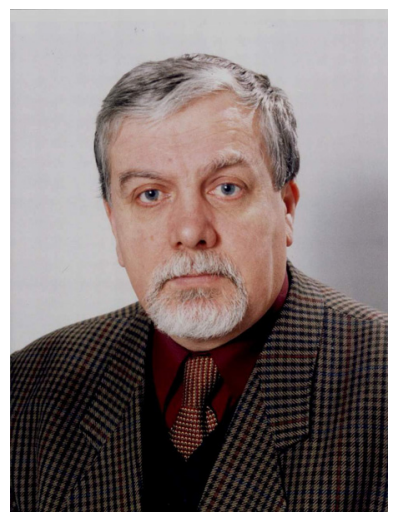

Petro Stakhiv, Ph.D., D. Sc., Professor born in 1948 in Lviv region, Ukraine. $\mathrm{He}$ graduated from Physical Faculty of Lviv State University in 1970 and received the M.Sc. degree in radiophysics and electronics. Since 1970 till 1973 he was a Ph.D. student at Department of Theoretical Fundamentals of Electro and Radiotechnics. In 1975 he received the Ph.D. degree in theoretical electrical engineering. The theme of his Ph.D. work was "Synthesis of linear electric circuits (method of state variables)». In 1992 he received D.Sc. degree in the same specialty after doctor thesis defend on "Analysis of dynamic regimes in electric and electronic circuits with multipolar elements». Since 
1973 till 1996 he was assistant, associate professor, professor and Head of Department of Theoretical Fundamentals of Electro and Radiotechnics at Lviv State University. In 1996 he began working at Lviv Polytechnic National University as professor and Head of Department of Theoretical and Electrical Engineering. Since 2003 he is an associate professor in the Technical University of Łódź.

His scientific interests are mainly concerned with mathematical modeling and simulation of dynamic processes in electrotechnical systems, numerical methods, methods of optimization, system theory and parallel programming.

He proposed different numerical procedures for calculation of dynamic processes on the basis of diacoptic approach. He developed an original method for discrete mathematical model synthesis of multipolar systems using optimization.

He is the author and co-author of more than 150 publications including 4 monographies, articles and conference proceedings.

Under his supervising 10 Ph.D. and 1 D.Sc. dissertations were prepared. He is an IEEE member and member of Editorial Boards of 2 scientific journals. 